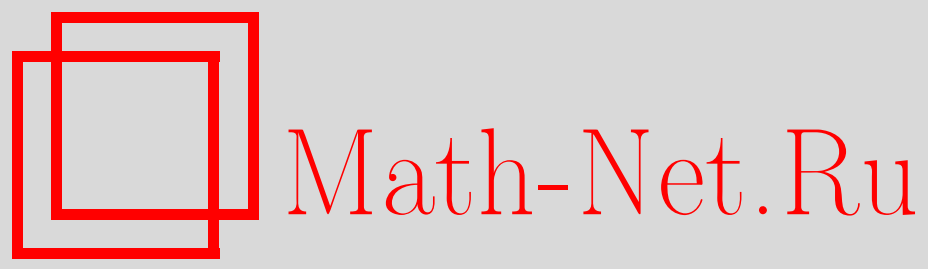

Г. М. Губреев, Безусловные базисы гильбертовых пространств, составленные из значений целых вектор-функций экспоненциального типа, Функи. анализ и его прил., 1999, том 33, выпуск 1, 62-65

DOI: https://doi.org/10.4213/faa339

Использование Общероссийского математического портала MathNet.Ru подразумевает, что вы прочитали и согласны с пользовательским соглашением

http://www . mathnet.ru/rus/agreement

Параметры загрузки:

IP : 54.224 .60 .19

26 апреля 2023 г., 17:19:32

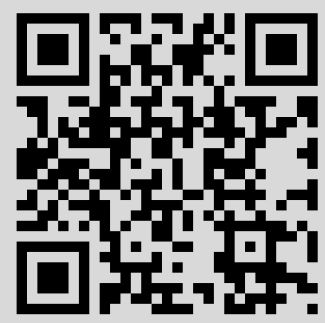




\title{
Безусловные базисы гильбертовых пространств, составленные из значений целых вектор-функций экспоненциального типа
}

\author{
(C) 1999. Г. М. ГУБРЕеВ
}

Успехи в исследовании базисных свойств конкретных семейств функций в пространствах $L_{2}(0, a)$ [1-3] привели к возможности изучения специальных классов безусловных базисов, ассоциированных с диссипативными вольтерровыми операторами, действующими в абстрактных гильбертовых пространствах [4]. Настоящая заметка посвящена изложению некоторых дальнейших результатов исследования, начатого в работе [4].

Пусть $e: \mathbb{C} \rightarrow \mathfrak{H}$ - целая функция экспоненциального типа со значениями в сепарабельном гильбертовом пространстве $\mathfrak{H}$ и $\tilde{e}(\lambda) \stackrel{\text { def }}{=} \lambda^{-1}(e(\lambda)-e(0))$. Такую функцию будем называть квазиэкспонентой, если выполнены следующие условия:

1) для каждого $z \in \mathbb{C}$ существует такой вектор $h(z) \in \mathfrak{H}$, что

$$
(\tilde{e}(\lambda), e(z))=(e(\lambda), h(z))
$$

при всех $\lambda \in \mathbb{C}$;

2) $\operatorname{Im}\left(\sum_{k} c_{k} \tilde{e}\left(\lambda_{k}\right), \sum_{k} c_{k} e\left(\lambda_{k}\right)\right) \geqslant 0$ для любых конечных совокупностей комплексных чисел $\left\{c_{k}\right\}_{1}^{n}$ и $\left\{\lambda_{k}\right\}_{1}^{n}$, причем $\operatorname{Im} \lambda_{k}>0, k=1, \ldots, n$.

Далее, квазиэкспоненту е будем называть регулярной, если существует хотя бы одна такая последовательность $\left\{\lambda_{n}\right\}_{-\infty}^{+\infty}, \inf _{k} \operatorname{Im} \lambda_{k}>0$, что семейство $\left\{e\left(\lambda_{n}\right)\right\}_{-\infty}^{+\infty}$ образует безусловный базис пространства $\mathfrak{H}$.

Задача об описании базисов, состояших из значений квазиэкспонент, распадается на две части. Необходимо, во-первых, описать все регулярные квазиэкспоненты и, во-вторых, дать описание всех безусловных базисов пространства $\mathfrak{H}$, составленных из значений регулярной квазиэкспоненты.

Обозначим через $\Lambda^{\text {(ехр) }}[5]$ класс всех вольтерровых диссипативных операторов $B$ в пространстве $\mathfrak{H}$, для которых резольвента $(I-\lambda B)^{-1}$ имеет конечный экспоненциальный тип. Мы получим пример квазиэкспоненты, если положим

$$
e(\lambda)=(I-\lambda B)^{-1} y,
$$

где $y-$ произвольный элемент из $\mathfrak{H}$. В самом деле,

$$
(\tilde{e}(\lambda), e(z))=\left(e(\lambda), B^{*} e(z)\right),
$$

$$
\operatorname{Im}\left(\sum_{k} c_{k} \tilde{e}\left(\lambda_{k}\right), \sum_{k} c_{k} e\left(\lambda_{k}\right)\right)=\frac{1}{2 i}\left(\left(B-B^{*}\right) \sum c_{k} e\left(\lambda_{k}\right), \sum c_{k} e\left(\lambda_{k}\right)\right) \geqslant 0 .
$$

*Эта работа выполнена при поддержке Международного научного фонда, гранты UCZ000, UCZ200. 
В частности, квазиэкспонентой в $L_{2}(0, a)$ является вектор-функция

$$
e(\lambda)=\frac{d}{d x} \int_{0}^{x} y(x-t) e^{i \lambda t} d t, \quad y \in L_{2}(0, a),
$$

отвечающая выбору $\mathfrak{H}=L_{2}(0, a), B=J_{a}$, где

$$
\left(J_{a} f\right)(x)=i \int_{0}^{x} f(t) d t .
$$

Особенно важную роль играют квазиэкспоненты этого класса, которые с помощью изложенной ниже процедуры порождаются весами $w^{2}$, удовлетворяющими условию Макенхаупта

$$
\sup \left\{|K|^{-1} \int_{K} w^{2}(x) d x|K|^{-1} \int_{K} w^{-2}(x) d x\right\}<\infty,
$$

где $K$ - произвольный интервал вещественной оси, а $|K|$ - его длина. Для каждого такого веса существует внешняя в области $\operatorname{Im} \lambda<0$ функция $w_{-}$, такая, что $\left|w_{-}(x-i 0)\right|^{2} \stackrel{\text { п.в. }}{=} w^{2}(x), x \in \mathbb{R}$. Эта функция допускает интегральное представление [3]:

$$
w_{-}(\lambda)=\lambda \int_{0}^{\infty} e^{-i \lambda t} y_{w}(t) d t, \quad \operatorname{Im} \lambda<0,
$$

с некоторой функцией $y_{w} \in L_{2}^{\text {loc }}\left(\mathbb{R}_{+}\right)$. Обозначим теперь через $e_{w}$ квазиэкспоненту вида $(2)$ с $y(t) \stackrel{\text { п.в. }}{=} y_{w}(t), t \in[0, a]$. Отметим, что квазиэкспоненты $e_{w}$ регулярны [3].

Оказывается, что регулярные квазиэкспоненты следует искать в классе вектор-функций вида (1).

ТЕОРемА 1. Каждая регулярная квазиэкспонента $е: \mathbb{C} \rightarrow \mathfrak{H}$ допускает представление

$$
e(\lambda)=(I-\lambda B)^{-1} y, \quad y \in \mathfrak{H},
$$

где $B \in \Lambda^{(\exp )}$ имеет тривиальное ядро и ядерную мнимую компоненту.

Две квазиэкспоненты $e_{i}$ со значениями в пространствах $\mathfrak{H}_{i}(i=1,2)$ назовем эквивалентными, если существует непрерывный и непрерывно обратимый оператор $S$ из $\mathfrak{H}_{1}$ на $\mathfrak{H}_{2}$, такой, что $S e_{1}(\lambda)=e_{2}(\lambda)$ для всех $\lambda \in \mathbb{C}$. Ясно, что эквивалентные квазиэкспоненты могут быть регулярными лишь одновременно. Основным результатом заметки является

Теорема 2. Квазиэкспонента $е: \mathbb{C} \rightarrow \mathfrak{H}$, экспоненциальньй тип которой равен а, регулярна тогда и только тогда, когда выполнень следующие условия:

1) вес $w^{2}(x) \stackrel{\text { def }}{=}\|e(x)\|^{2}$ удовлетворяет условию $\left(\mathrm{A}_{2}\right)$ на $\mathbb{R}$;

2) е эквивалентна квазиэкспоненте $e_{w}$ со значениями в $L_{2}(0, a)$, построенной по весу $w^{2}$ на $\mathbb{R}$.

Таким образом, квазиэкспоненты $e_{w}$, где $w^{2}$ - произвольный вес Макенхаупта на $\mathbb{R}$, являются свободной моделью в классе всех регулярных квазиэкспонент со значениями в абстрактных гильбертовых пространствах. Отметим также, что условие эквивалентности квазиэкспонент $e$ и $e_{w}$ равносильно 
равенствам

$$
B=S^{-1} J_{a} S, \quad S y=y_{w}, \quad S: \mathfrak{H} \rightarrow L_{2}(0, a),
$$

где оператор $B$ и вектор $y$ отвечают квазиэкспоненте $e$ в силу теоремы 1. Поэтому теорема 2 может быть положена в основу нового метода доказательства критериев подобия вольтерровых диссипативных операторов. Первоначальные результаты в этом направлении получены в [4]; дальнейшему их развитию будет посвящена отдельная статья.

Поскольку центральной частью теоремы 2 является необходимость условия 1), то наметим основные вехи в доказательстве этого утверждения. Прежде всего, для регулярных квазиэкспонент е устанавливается сходимость интегралов

$$
\int_{-\infty}^{+\infty} w^{ \pm 2}(x)\left(1+x^{2}\right)^{-1} d x, \quad w(x)=\|e(x)\| .
$$

Поэтому сушествует внешняя в области $\operatorname{Im} \lambda>0$ функция $w_{+}$, для которой $\left|w_{+}(x+i 0)\right|^{2} \stackrel{\text { п.в. }}{=} w^{2}(x), x \in \mathbb{R}$. Доказывается, что $\|e(\lambda)\|<\left|w_{+}(\lambda)\right|$ при $\operatorname{Im} \lambda>0$. Второй шаг состоит в том, чтобы, отправляясь от этого неравенства, получить оценку для интеграла Пуассона

$$
\frac{\operatorname{Im} \lambda}{\pi} \int_{-\infty}^{+\infty} w^{2}(x)|x-\lambda|^{-2} d x \leqslant K\left|w_{+}(\lambda)\right|^{2}, \quad \operatorname{Im} \lambda>0 .
$$

Далее, модифицируя соответствующие рассуждения из [4], доказываем ограниченность оператора $w \mathscr{H} w^{-1}-w \varphi^{-1} \mathscr{H} \varphi w^{-1}$ в пространстве $L_{2}(\mathbb{R})$. $3_{\text {десь }}$ $\mathscr{H}$ — преобразование Гильберта, а

$$
\varphi(\lambda)=e^{i a \lambda / 2} \mathrm{v} \cdot \mathrm{p} \cdot \prod_{k}\left(1-\frac{\lambda}{\lambda_{k}}\right),
$$

где $\left\{\lambda_{k}\right\}_{-\infty}^{+\infty}$ - такая последовательность, что семейство $\left\{e\left(\lambda_{k}\right)\right\}_{-\infty}^{+\infty}$ образует безусловный базис в $\mathfrak{H}$. Записывая условие ограниченности этого оператора на функциях $(x-\lambda)^{-1}, \operatorname{Im} \lambda \neq 0$, и учитывая $(4)$, приходим к ограниченности оператора $w \mathscr{H} w^{-1}$, что и требовалось.

Перейдем теперь к описанию безусловных базисов, порожденных регулярной квазиэкспонентой. Соединяя сказанное выше с результатами из [3], приходим к следующей теореме.

ТЕОРема 3. Пусть $е: \mathbb{C} \rightarrow \mathfrak{H}$ - регулярная квазиэкспонента экспоненииального типа а $и w(x) \stackrel{\text { def }}{=}\|e(x)\|, x \in \mathbb{R}$. Тогда семейство $\left\{e\left(\lambda_{k}\right)\right\}_{-\infty}^{+\infty}$, $\inf _{k} \operatorname{Im} \lambda_{k}>0$, образует безусловньй базис пространства $\mathfrak{H}$ в том и только том случае, когда последовательность $\left\{\lambda_{k}\right\}_{-\infty}^{+\infty}$ совпадает с множеством (простьх) корней целой функции конечной степени $\varphi$, удовлетворяющей условиям

1) $h_{\varphi}(\pi / 2)=0, h_{\varphi}(-\pi / 2)=a$;

2) вес $w^{-2}(x)|\varphi(x)|^{2}$ удовлетворлет условию $\left(\mathrm{A}_{2}\right)$ на $\mathbb{R}$;

3) последовательность $\left\{\lambda_{k}\right\}_{-\infty}^{+\infty}$ отделима в смьлле Карлесона, т.е.

$$
\inf _{k} \prod_{j \neq k}\left|\lambda_{k}-\lambda_{j}\right| \cdot\left|\lambda_{k}-\bar{\lambda}_{j}\right|^{-1}>0
$$


Не вдаваясь в подробности, отметим, что аналогичный результат можно сформулировать и без ограничения $\inf _{k} \operatorname{Im} \lambda_{k}>0$.

В качестве приложения сформулированных результатов дадим описание всех регулярных квазиэкспонент вида (2). Для этого рассмотрим множество всех аналитических в области $\operatorname{Im} \lambda<0$ функций вида $W(\lambda)=S(\lambda) V(\lambda)$, где функция $V$ внешняя, причем вес $|V(x-i 0)|^{2}$ удовлетворяет условию $\left(\mathrm{A}_{2}\right)$ на $\mathbb{R}$, а $S$ внутренняя и такая, что

$$
\inf _{\operatorname{Im} \lambda<0}\{|S(\lambda)|+\exp (\operatorname{Im} \lambda a)\}>0 .
$$

Каждая такая функция $W$ изображается в виде

$$
W(\lambda)=\lambda \int_{0}^{\infty} e^{-i \lambda t} y_{W}(t) d t, \quad \operatorname{Im} \lambda<0, y_{W} \in L_{2}^{\operatorname{loc}}\left(\mathbb{R}_{+}\right) .
$$

Полученное таким образом множество функций из $L_{2}^{\text {loc }}\left(\mathbb{R}_{+}\right)$обозначим через $\mathscr{M}$.

ТеоремА 4. Квазиэкспонента вида (2) со значениями в $L_{2}(0, a)$ регулярна тогда и только тогда, когда у почти всюду совпадает с сужением на $[0, a]$ некоторой функиии $y_{W} \in \mathscr{M}$.

Доказательство основано на теореме Сарасона о коммутанте [6], поскольку в этом случае $B=J_{a}$ и равенство (3) означает, что $S$ принадлежит коммутанту $J_{a}$. Из теоремы о короне [7] выводится, что условие (5) равносильно непрерывной обратимости оператора $S$.

Теорема 3 дает описание всех безусловных базисов, порожденных такими квазиэкспонентами. В частности, имеет место

СлЕдСТВИЕ. Семейство функиий

$$
\frac{d}{d x} \int_{0}^{x} y(x-t) e^{i n t} d t, \quad n \in \mathbb{Z},
$$

образует безусловньий базис в $L_{2}(0,2 \pi)$ тогда и только тогда, когда $у$ почти всюду совпадает с сужением на $[0, a]$ некоторой функиии $y_{W} \in \mathscr{M}$.

\section{ЛитЕРАТУРА}

1. Hruscev S. V., Nikol'skii N. K., Pavlov B. S. Lect. Notes in Math., Vol. 864, 1981, pp. 214-335. 2. Губреев Г. М. Изв. АН АрмССР, Математика, 23, № 3, 237-269 (1988). 3. Губреев Г. М. Записки научн. сем. ЛОМИ, Исследования по линейным операторам и теории функций, 19, 190, 34-80 (1991). 4. Губреев Г. М. Матем. сборник, 183, № 9, 105-146 (1992). 5. Бродский $M$. C. Треугольные и жордановы представления линейных операторов. Наука, М., 1969. 6. Sarason D. Trans. Amer. Math. Soc., 127, No. 2, 179-203 (1967). 7. Гофман K. Банаховы пространства аналитических функций. ИЛ, М., 1963.

Южноукраинский педагогический 